\title{
Hold Up: Modelo de Detecção e Controle de emoções em Ambientes Acadêmicos
}

\author{
Gabriel Di Iorio, Victor Ströele, Mário Dantas, Fernanda Campos \\ ${ }^{1}$ Computer Science Postgraduate Program - Federal University of Juiz de Fora \\ 36036-900- Juiz de Fora- MG- Brazil \\ \{gabriel.iorio, victor.stroele, mario.dantas\}@ice.ufjf.br, \\ fernanda.campos@ufjf.edu.br
}

\begin{abstract}
Stress and anxiety are factors that affect student's performance. The Hold Up model seeks to assist the student in detecting and controlling emotions in order to improve ther school performance. The model detects heart rate oscillations through sensors and defines the student's emotional profile and context. Variations in heart rate may indicate stress situations that, when uncontrolled, impact student's performance during school activity. The proposal was evaluated with students from a Brazilian university. The results point to the feasibility of the proposal and the comments provided positive indications that this approach can be used in educational environments.
\end{abstract}

Resumo. Estresse e ansiedade são fatores que afetam o desempenho do aluno. O modelo Hold Up busca auxiliar o aluno na detecção e controle das emoções, visando melhorar seu desempenho acadêmico. O modelo detecta oscilações na frequência cardíaca por meio de sensores e define o perfil e contexto emocional do aluno. Variações na frequência cardíaca podem indicar situações de estresse que, quando não controladas, prejudicam o desempenho do aluno durante a realização da atividade acadêmica. A proposta foi avaliada com alunos de uma universidade brasileira. Os resultados apontam a viabilidade da proposta e os comentários opinativos forneceram indícios positivos de que esta abordagem pode ser usada em ambientes educacionais.

\section{Introdução}

Estudos apontam a importância de se manter atento, calmo e concentrado para uma melhor compreensão do conteúdo durante o processo de ensino e aprendizagem. Alunos que se encontram em situação de estresse apresentam dificuldades para absorver o conteúdo apresentado e expressar o seu próprio conhecimento [Mondardo and Pedon 2005]. Desta forma, é fundamental que os alunos consigam equilibrar suas emoções em momentos de adversidade e nos momentos de avaliação e apresentação em público, visto que isso impacta diretamente na sua aprendizagem [Hanceroglu 2017].

Além disso, outras pesquisas direcionadas à educação relatam também a influência do estresse e ansiedade no ambiente acadêmico [Lee et al. 2018]. Tal questão pode levar o aluno a um baixo desempenho e doenças como depressão [Beiter et al. 2015] devido, por muitas vezes, às avaliações que podem ser fatores determinantes no agravamento dos casos. Áreas como a medicina, psicologia, psicopedagogia, entre outras, tratam desse 
VIII Congresso Brasileiro de Informática na Educação (CBIE 2019)

Anais do XXX Simpósio Brasileiro de Informática na Educação (SBIE 2019)

tema, porém, no âmbito tecnológico, poucas são as abordagens que visam identificar essa adversidade.

Por outro lado, as constantes mudanças decorridas do avanço científico e tecnológico estão gerando impactos em diversas camadas da sociedade, afetando o dia a dia das pessoas e empresas. Nesse cenário, surge o termo Transformação Digital (TD), referente ao processo em que uma empresa implementa uma mentalidade digital em todos os seus setores. Inicialmente a TD estava relacionada a mudanças no setor empresarial, mas já é observada em outras esferas. [Bradley et al. 2015] apresentam um estudo no qual é evidente o impacto da Transformação Digital na educação. O vortex da Transformação Digital nos mostra que a educação ocupa a sexta colocação dentre os domínios que estão sofrendo os maiores impactos tecnológicos até 2020. Logo, assim como enquadramentos empresariais se alteram perante tais mudanças tecnológicas, o setor educacional também procura se moldar para novas demandas.

Constantemente é observado em pesquisas no domínio educacional, a importância do uso das tecnologias e como as mesmas se apresentam como uma nova tendência na revolução das práticas educacionais. Tal mudança pode ser evidenciada em trabalhos como [Lopes et al. 2018] e [Dantas et al. 2018] que relatam o impacto das tecnologias nas smart classrooms, que são baseadas em Internet das Coisas ou IoT (Iternet of Things).

A Internet das Coisas exprime a ideia de uma rede de objetos capaz de compartilhar informações, dados e recursos, agindo e reagindo conforme as mudanças no ambiente [Madakam 2015]. A IoT aplicada na educação gera uma nova gama de possibilidades que devem ser exploradas num cenário propício para propagação de tecnologias transformadoras.

No contexto deste trabalho, seguindo as diretrizes da Transformação Digital, utilizaremos IoT para a detecção e controle de ansiedade e nervosismo em ambientes acadêmicos. Durante o processo de formação, os alunos passam por períodos de angústia e apreensão, uma vez que esse processo representa uma fase de grande mudança na vida das pessoas como forma de preparação dos indivíduos para uma carreira profissional. Circunstâncias essas que fazem com que os mesmos não tenham o desempenho esperado, por exemplo, em avaliações, apresentações de trabalhos e reuniões [Mondardo and Pedon 2005].

Com o intuito de unir as necessidades educacionais dos alunos e a transformação digital na educação, propomos o modelo Hold Up, capaz de monitorar os alunos, através do uso de sensores, para definição do seu perfil e contexto emocional. Os dados referentes à frequência cardíaca dos alunos são coletados e analisados possibilitando que o modelo identifique se o aluno se encontra em situações de estresse, nervosismo ou ansiedade. Com base nessas informações são recomendadas ações ao aluno com o intuito de ajudálo a controlar suas emoções e retomar a concentração necessária para obter sucesso no desenvolvimento da atividade.

A questão que se coloca como pesquisa é: o modelo Hold Up é capaz de monitorar e detectar que o aluno se encontra em um momento de estresse, nervosismo ou ansiedade, e apresentar notificações para que ele saia da condição em que se encontra? Neste sentido, este artigo apresenta o modelo proposto e a condução de um estudo de viabilidade onde o uso do modelo Hold Up é avaliado, em um ambiente real com alunos voluntários 
VIII Congresso Brasileiro de Informática na Educação (CBIE 2019)

Anais do XXX Simpósio Brasileiro de Informática na Educação (SBIE 2019)

de um curso de graduação em Ciência da Computação de uma universidade brasileira.

Em síntese, como contribuições podemos destacar que este trabalho (I) considera os efeitos da transformação digital na educação (II) fazendo uso de IoT (III) para auxiliar os alunos no controle de suas emoções, (IV) buscando a melhora no seu desempenho acadêmico.

O trabalho está organizado na seguinte sequência: na Seção 2 são apresentados os trabalhos correlatos, na Seção 3 é apresentado o modelo Hold Up; na Seção 4 um estudo de viabilidade é conduzido e, na Seção 5 são feitas as considerações finais e os trabalhos futuros.

\section{Trabalhos Relacionados}

Inicialmente, podemos observar o impacto já presente que a computação agrega à educação. Como o observado na pesquisa feita por [Sung et al. 2016] que apresenta um estudo a respeito da integração de dispositivos móveis na educação, analisando também suas vantagens e desvantagens, tomando como base artigos publicados entre 1993 e 2013. Tecnologias como Handhelds, smartphones e laptops geraram a maior proporção dos estudos identificados pelo trabalho. Assim, em um primeiro momento, verificou-se que poucas pesquisas abordam o uso de dispositivos móveis, como sensores, na educação.

Ademais, foi observado o uso do smartwatch no auxílio de alunos com Deficiência Intelectual no trabalho feito por [Zheng and Genaro Motti 2018]. Esse trabalho consiste no envio de notificações como parabenizações para indivíduos participativos, avisos para que os mesmos não desviem o foco e mensagens de incitação a laboração para esses usuários. Logo, os mesmos são encorajados a participar de forma ativa nas aulas para que assim não fiquem exclusos. Tal propositura faz uso de tecnologias similares à proposta neste artigo, porém para um fim diferente.

[Mota et al. 2018] apresentam um processo de aprendizagem ubíqua, relacionada à educação ambiental por meio de mensagens motivacionais. Assim, conseguem potencializar a motivação e aprendizagem por meio da teoria da autodeterminação. Também foram identificados trabalhos que abordam a melhora da atenção dos alunos por meio da tutoria mindfulness em sistemas tutores inteligentes [Alves et al. 2018]. Segundo os autores, através de exercícios respiratórios, é possível aprimorar a atenção do aluno e, consequentemente, sua atenção na tarefa realizada.

Diferentemente dos trabalhos supracitados, esse trabalho se propõe a realizar uma junção dessas duas grandes frentes: monitoramento de alunos e auxílio no processo de aprendizagem por meio do uso de tecnologias. A coleta dos dados a respeito da frequência cardíaca nos permite realizar inferências sobre o usuário, como se o mesmo está dormindo, estressado ou ansioso. Assim, o paradigma da aprendizagem ubíqua pode ser adotado no intuito monitorar o aluno e sugerir, por meio de notificações, que ele realize determinada ação a fim de controlar o seu estado emocional e melhorar seu desempenho em uma situação de estresse.

\section{Hold Up - Um modelo de recomendação baseado em IoT}

Este trabalho descreve o modelo de recomendação Hold Up, para auxiliar no controle das emoções e no aprimoramento do desempenho de alunos em um ambiente acadêmico. $\mathrm{O}$ 
VIII Congresso Brasileiro de Informática na Educação (CBIE 2019)

Anais do XXX Simpósio Brasileiro de Informática na Educação (SBIE 2019)

modelo é preparado para detectar oscilações na frequência cardíaca por meio de sensores e, assim, reconhecer o perfil e contexto no qual o aluno se insere. Observar variações de frequência cardíaca se mostra interessante, pois, indicam situações de estresse que podem acarretar maus resultados quando não contornados devido ao nervosismo em ocasiões importantes. A Figura 1 apresenta uma visão geral do modelo proposto.

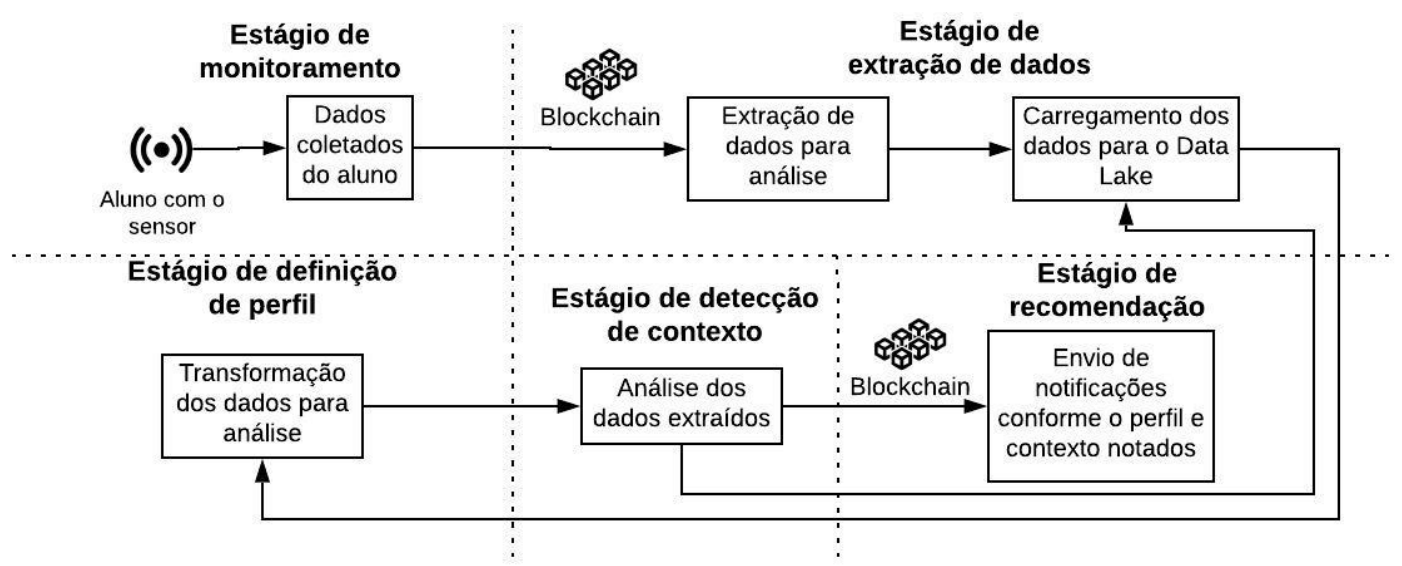

Figura 1. Visão Geral do modelo Hold Up

O estágio de monitoramento é responsável por monitorar os alunos no ambiente acadêmico. O modelo foi projetado para que seja possível o uso de diferentes tipos de sensores. Neste trabalho, para a coleta dos dados, foram utilizadas smartbands, pulseiras inteligentes utilizadas como sensores para o monitoramento e coleta de dados de frequência cardíaca e de sono.

O uso de sensores gera a necessidade de um estágio de extração de dados capaz de receber, processar e armazenar os dados enviados por eles. Assim, o estágio de extração é responsável por "escutar" os sensores e armazenar os dados coletados em um banco de dados. Visto que o monitoramento pode gerar um grande volume de dados, o estágio de extração armazena os dados em um banco de dados NoSQL, seguindo os conceitos de Data Lake [Inmon 2016]. Dessa forma, temos a garantia de que todos os dados dos alunos enviados pelos sensores no estágio de monitoramento serão armazenados, evitando perdas que podem impactar no funcionamento do modelo.

O estágio de definição de perfil é responsável por caracterizar cada aluno segundo o seu histórico de batimentos cardíacos para que seja possível, em momentos posteriores, identificar possíveis oscilações no mesmo. O perfil pode ser definido de maneira explícita ou implícita. Com os dados do monitoramento armazenados, é feita a transformação e consolidação desses dados para a definição do perfil implícito de cada aluno. Nesta etapa, é feita uma análise descritiva dos batimentos cardíacos e os outliers são descartados para evitar que valores muito altos ou muito baixos afetem a média e, consequentemente, o perfil cardíaco do aluno. O modelo também permite que o aluno informe a sua frequência cardíaca média e, neste caso, o perfil é definido explicitamente.

O estágio de detecção de contexto é responsável por identificar o estado emocional do aluno, verificando se ele se encontra em uma situação de estresse, ansiedade ou 
nervosismo. Neste estágio é feita a análise dos dados enviados pelo estágio de monitoramento e dos dados de perfil do aluno para verificar se há uma oscilação na medição. Como avalia-se os dados de frequência cardíaca e movimentação, foi feita uma pesquisa acerca dos dados normais de frequência cardíaca em situações de repouso e de atividade física. Com essa análise, constatou-se que algumas variáveis podem ser descartadas durante a avaliação dos dados, como o peso do usuário. Trabalhos como [Subramaniam 2011] relatam que o IMC (Índice de Massa Corporal) tem pouca influência na frequência cardíaca. A idade também é um dos fatores que leva a mudanças significativas na frequência cardíaca. Entretanto, como, inicialmente, este projeto visa dar assistência para alunos de graduação, a idade padrão considerada foi de 17 a 30 anos. Isto posto, também foi identificado que a frequência cardíaca normal de um indivíduo, nesta faixa etária, varia entre $60 \mathrm{bpm}$ a $100 \mathrm{bpm}$ (batimentos por minuto) ${ }^{1}$.

Outro fator importante a ser apontado é que a grande maioria dos sensores de monitoramento que fazem a medição de frequência cardíaca, possuem acelerômetro. Logo, caso seja identificado que o usuário está em movimento constante e sua frequência seja monitorada e constatada como alta, o modelo não envia uma notificação, dado que, provavelmente, o mesmo não está em situação de estresse e, provavelmente, realizando alguma atividade física. Evitando assim, recomendações desnecessárias.

Além disso, com os dados obtidos pela smartband a respeito da frequência cardíaca, conseguimos inferir se um aluno está com as horas diárias de sono conforme o recomendado pelos órgão de saúde, sendo de 7 a 9 horas diárias o ideal para alunos na faixa etária deste estudo ${ }^{2}$. Tal medição é importante pois se relaciona diretamente com o estresse e, por consequência, com sua performance acadêmica [Gaultney 2010]. Munido dessas informações somos capazes de indicar para o aluno que suas horas de sono estão abaixo do recomendado e que isso causa resultados negativos no seu desempenho acadêmico.

Caso seja detectado que o aluno se encontra em um contexto de estresse, ansiedade ou nervosismo, o estágio de recomendação apresenta uma mensagem a ele com o intuito de ajudá-lo a sair dessa condição. As possíveis recomendações a serem enviadas podem ser divididas em três grupos de acordo com a gama de recomendações possíveis para o aluno na proposta desse trabalho: atividades imediatas, atividades preventivas e indicações para qualidade de sono. É importante destacar que a divisão nesses três grupos de atividades foi feita a fim de viabilizar o estudo aqui realizado.

As atividades imediatas são aquelas possíveis de serem realizadas em qualquer momento, sem a necessidade de um espaço físico apropriado. Assim, caso o aluno esteja presente em um cenário como uma avaliação, apresentação ou reunião, tais medidas são eficazes uma vez que são de fácil realização e rápidas. As atividades imediatas adotadas neste trabalho são:

- Brain Breaks: consiste em realizar uma pausa na atividade que lhe causa ansiedade e fazer alguma atividade prazerosa, cantar uma música de seu gosto ou simplesmente interromper por alguns minutos a ação que gera estresse ${ }^{3}$.

\footnotetext{
${ }^{1}$ www.heart.org

${ }^{2}$ www.sleepfoundation.org

${ }^{3}$ www.edutopia.org
} 
VIII Congresso Brasileiro de Informática na Educação (CBIE 2019)

Anais do XXX Simpósio Brasileiro de Informática na Educação (SBIE 2019)

- Respiração Diafragmática: consiste em efetuar exercícios respiratórios que tem como principal característica a troca de todo o ar dos pulmões [de Oliveira and Duarte 2004].

- Relaxamento Muscular Progressivo: consiste numa série de exercícios simples e fáceis que ajudam no relaxamento da musculatura para redução nos níveis de ansiedade [de Oliveira and Duarte 2004].

- Auto-conversa positiva: consiste em conversar consigo mesmo com frases motivadoras e que auxiliem no encorajamento ${ }^{4}$.

Vale ressaltar que as atividades imediatas não são mutuamente exclusivas, ou seja, os alunos podem realizar mais de uma ao mesmo tempo. Caso ele esteja, por exemplo, iniciando uma avaliação, ele pode fazer uma pausa (Brain Breaks) e a respiração diafragmática de forma conjugada.

As atividades preventivas são aquelas com o objetivo de evitar a ocorrência de casos mais graves de picos de frequência cardíaca, como taquicardia ou recorrência de grandes oscilações na frequência diversas vezes ao dia. Para que o aluno faça essas atividades é necessário que ele esteja em um ambiente apropriado. São elas:

- Atividades Físicas: consiste em realizar atividades físicas aeróbicas a fim de aumentar propositalmente a frequência cardíaca por um período prolongado de tempo para que assim, seja lançada endorfina na corrente sanguínea, provocando um relaxamento depois que o exercício é finalizado.

- Relaxamento ao ar livre: consiste em ir para lugares com um contato direto com a natureza para que assim o aluno consiga relaxar.

- Procura de ajuda médica: em alguns casos, é interessante que o usuário vá a um médico ou terapeuta uma vez que essas ações podem não ter o efeito esperado.

As indicações para qualidade de sono dizem respeito às recomendações feitas pelo modelo Hold Up para que o aluno obtenha de 7 a 9 horas de sono. Assim, conforme o montante total de horas de sono identificado pelo sensor que o usuário está usando, seremos capazes de indicar a necessidade de que o mesmo durma mais cedo. Dessa forma, o aluno terá uma consequente redução nos possíveis picos de estresse e, por conseguinte, uma melhora em seu rendimento acadêmico.

Uma ressalva válida é que a segurança e confiabilidade dos dados é garantida em dois momentos, seguindo o conceito de Blockchain: durante o envio dos dados coletados para o Data Lake e durante o envio de recomendações para o aluno. Dessa forma, dificulta-se a adulteração e acesso dos dados do aluno por indivíduos não autorizados, sendo necessária a quebra da criptografia da Blockchain.

\section{Estudo de Viabilidade}

A avaliação do modelo Hold Up é descritiva, feita através de um Estudo de Viabilidade. O objetivo principal de um estudo de viabilidade é criar uma quantidade suficiente de conhecimento sobre a solução proposta, buscando responder a uma questão de pesquisa [Lacerda et al. 2015] através das seguintes etapas: definição, formulação do objetivo, planejamento, execução/observação e apresentação dos resultados. No contexto educacional

\footnotetext{
${ }^{4}$ www.heart.org
} 
um estudo de viabilidade exige a descrição completa de uma atividade, com diferentes situações iniciadas e finalizadas através de eventos, resultado de ações do aluno sobre o ambiente educacional ou vindas do próprio ambiente. Para responder a questão de pesquisa inicialmente descrita, o modelo proposto foi aplicado em um ambiente real de aprendizagem, em momentos nos quais os alunos se encontravam, possivelmente, em situações de estresse. Mostrou-se também o contexto de cada aluno e as possíveis notificações a serem realizadas.

O estudo de viabilidade foi realizado com alunos voluntários de um curso de graduação em Ciência da Computação de uma universidade brasileira. Os alunos foram monitorados durante a execução de um dos três tipos de atividades acadêmicas: apresentação de trabalho, avaliações escritas e reuniões de orientação em trabalhos de conclusão de curso.Foram realizadas notificações considerando os tipos de atividades a serem recomendadas e o contexto emocional do aluno identificado pelo modelo Hold Up.

Para a definição do perfil dos alunos foi necessário que cada um deles aferisse sua frequência cardíaca inicialmente, em uma situação de repouso, para determinarmos qual é sua frequência cardíaca normal quando eles não se encontravam em situações de estresse. Após as medições, calculou-se a frequência cardíaca média dos alunos envolvidos neste estudo para que assim seus perfis fossem definidos em situações de repouso. Logo, com todos os alunos examinados nesse estudo, identificou-se que a frequência cardíaca média em situações de repouso ficava na faixa de $60 \mathrm{bpm}$ a $70 \mathrm{bpm}$ entre todos os participantes. Após a definição do perfil, os alunos foram monitorados durante a execução de atividades acadêmicas e a etapa de execução deste estudo de viabilidade seguiu os passos descritos na Figura 2.

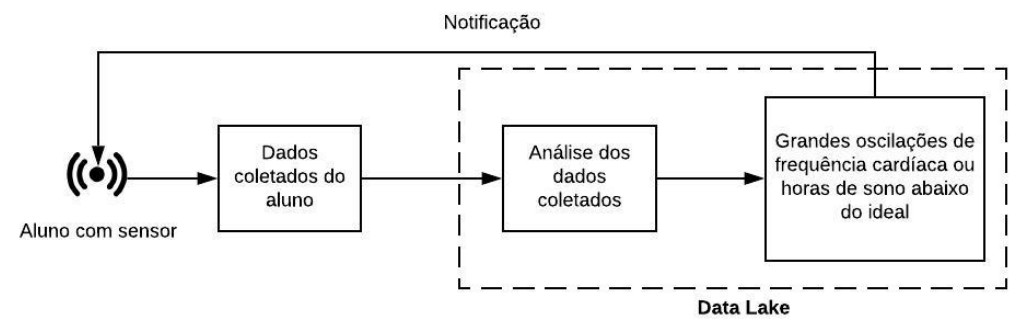

Figura 2. Etapas de execução.

Os dados coletados dos alunos através das smartband foram enviados para o MongoDB, por ser um banco de dados NoSQL amplamente utilizado em soluções Data Lake. Subsequentemente, esses dados foram analisados e o modelo Hold Up traçou o contexto dos alunos para identificar seus estados emocionais e verificar a necessidade de envio de alguma recomendação. Assim, os alunos que, em algum momento, apresentavam oscilações de frequência cardíaca ou que obtiveram uma quantidade de horas de sono abaixo do indicado, receberam uma notificação.

Os alunos foram monitorados durante as atividades acadêmicas. A Tabela 1 apresenta as frequências cardíacas de alguns dos alunos voluntários em três momentos da avaliação. Os números presentes na mesma se referem à primeira, segunda e terceira medição de frequência feitas, respectivamente. Percebe-se que, em geral, os alunos apre- 
VIII Congresso Brasileiro de Informática na Educação (CBIE 2019)

Anais do XXX Simpósio Brasileiro de Informática na Educação (SBIE 2019)

Tabela 1. Medições de frequência cardíaca e de sono

\begin{tabular}{|c|c|c|c|c|c|c|}
\hline \multirow{2}{*}{ Alunos } & \multicolumn{3}{|c|}{ Frequências } & \multicolumn{3}{|c|}{ Sono } \\
\hline & $1^{\circ}$ & $2^{\circ}$ & $3^{\circ}$ & Leve & Profundo & Total \\
\hline Aluno 1 & $83 \mathrm{bpm}$ & $103 \mathrm{bpm}$ & $78 \mathrm{bpm}$ & 1 h e $59 \mathrm{~min}$ & $4 \mathrm{~h}$ e $17 \mathrm{~min}$ & $6 \mathrm{~h}$ e $16 \mathrm{~min}$ \\
\hline Aluno 2 & $64 \mathrm{bpm}$ & 70 bpm & $68 \mathrm{bpm}$ & $3 \mathrm{he} 13 \mathrm{~min}$ & $4 \mathrm{~h} \mathrm{e} 43 \mathrm{~min}$ & $7 \mathrm{~h} \mathrm{e} 56 \mathrm{~min}$ \\
\hline Aluno 3 & $115 \mathrm{bpm}$ & $93 \mathrm{bpm}$ & $53 \mathrm{bpm}$ & $6 \mathrm{~h} \mathrm{e} 49 \mathrm{~min}$ & $2 \mathrm{~h}$ e $30 \mathrm{~min}$ & $8 \mathrm{~h} \mathrm{e} 19 \mathrm{~min}$ \\
\hline Aluno 4 & $61 \mathrm{bpm}$ & 88 bpm & $69 \mathrm{bpm}$ & 5 h e $24 \mathrm{~min}$ & $1 \mathrm{he} 17 \mathrm{~min}$ & $6 \mathrm{~h}$ e $41 \mathrm{~min}$ \\
\hline Aluno 5 & $68 \mathrm{bpm}$ & $98 \mathrm{bpm}$ & $72 \mathrm{bpm}$ & $1 \mathrm{~h} \mathrm{e} 36 \mathrm{~min}$ & 4 h e 51 min & $6 \mathrm{~h}$ e $27 \mathrm{~min}$ \\
\hline Aluno 6 & $70 \mathrm{bpm}$ & 101 bpm & $65 \mathrm{bpm}$ & 4 he $19 \mathrm{~min}$ & $4 \mathrm{he} 20 \mathrm{~min}$ & $8 \mathrm{~h}$ e $39 \mathrm{~min}$ \\
\hline
\end{tabular}

sentam um aumento na frequência cardíaca durante a atividade acadêmica, mas nem todos apresentaram oscilações, como o caso do Aluno 2. Isso é um indicativo de que alguns alunos permanecem calmos, mesmo diante de situações de possível ansiedade como provas, reuniões ou apresentações. Nestes contextos, o modelo não enviou notificações para o aluno.

Neste primeiro esforço de implementação, definiu-se que é pertinente enviar dois tipos de notificação aos usuários. Uma relacionada a atividades imediatas e outra a atividades preventivas visto que ambas fazem com que seu nível de estresse abaixe, cabendo assim ao usuário escolher qual delas melhor se adéqua ao ambiente em que o mesmo se encontra. Além disso, é meritório, também, informar o montante de horas de sono que o mesmo obteve na determinada noite e informá-lo caso o mesmo não tenha obtido o importe de horas necessárias.

Neste estudo, cerca de $20 \%$ dos alunos monitorados não receberam notificações, visto que o modelo não detectou que os mesmos se encontravam em situação de estresse. Por outro lado, aproximadamente $80 \%$ dos alunos receberam algum tipo de recomendação, seja para o controle emocional ou para a adequação das horas de sono.

Para verificar a aceitação da proposta por parte dos alunos e a adequação das recomendações foi enviado um formulário para resposta de todos os indivíduos que participaram na coleta de dados. No formulário constavam os dois grupos de recomendação a serem enviados: atividades imediatas e atividades preventivas. E, também, um breve comentário sobre o que consiste cada uma das atividades para redução de estresse. $\mathrm{O}$ formulário foi elaborado com questões como: "Você considera que a recomendação de uma atividade de cada grupo pode ajudar a reduzir seu nível de ansiedade e estresse?"; "As recomendações que lhe foram feitas foram adequadas e úteis para você?". As respostas das questões seguiram a escala Likert variando de 1 (péssimo) a 5 (ótimo). Os resultados são descritos em sequência.

Em síntese, identificou-se que $50 \%$ dos alunos observados qualificam a proposta do modelo Hold Up como sendo "ótima". Os 50\% restantes avaliaram a proposta como "boa". Logo, identifica-se que alguns dos alunos examinados avaliam a proposta como sendo "boa" devido à recomendações que não são úteis para os mesmos perante a situação por eles vivenciada, como a realização de atividades físicas durante uma prova. Os alunos 
VIII Congresso Brasileiro de Informática na Educação (CBIE 2019)

Anais do XXX Simpósio Brasileiro de Informática na Educação (SBIE 2019)

que não receberam recomendações não preencheram o formulário de avaliação. Por outro lado, através de entrevista informal, foram questionados se eles considerariam importante receber alguma notificação. Alguns deles relataram o interesse de receber dicas mais genéricas para o controle de emoções, como ouvir músicas.

\section{Considerações Finais e Trabalhos Futuros}

Este trabalho apresentou o modelo Hold $\boldsymbol{U p}$, capaz de identificar situações de estresse e ansiedade, além da qualidade do sono por meio de sensores e realizar recomendações para auxiliar no aprimoramento do desempenho acadêmico de alunos. Uma avaliação inicial da proposta por parte dos alunos examinados demonstra que a mesma é viável e desejada.

Algumas limitações foram encontradas durante a realização deste trabalho, tais como: o modelo de smartband usado no estudo, Xiaomi Mi Band 3, e seu software não são open source, ou seja, o código fonte do software usado nessa tecnologia não pode ser aplicado para diferentes fins. Apenas por engenharia reversa podemos realizar adaptações no software da mesma, tal questão não é interessante pois, por diversas vezes, isso pode comprometer a fluidez da aplicação. Além disso, vale ressaltar a quantidade de smartbands disponíveis para o estudo que fez com a coleta de dados por diversos alunos simultaneamente fosse impossibilitada.

Como trabalhos futuros pretende-se avaliar de forma mais incisiva os dados coletados a respeito do sono dos indivíduos para melhores recomendações. Outrossim, verificar a melhor forma de armazenamento, tratamento e segurança dos dados coletados é pertinente, visto que o grande volume de dados gerados pode torná-la lenta e inviável. Ademais, estudar o impacto das notificações recebidas pelos alunos é interessante uma vez que alguns alunos podem se sentir desconfortáveis com o envio das mesmas. Para mais, realizar a identificação emocional do aluno de forma mais abrangente de forma a se obter mais contextos possíveis para serem trabalhados além dos já avaliados como nervoso ou calmo.

\section{Agradecimentos}

Os autores gostariam de agradecer a FAPEMIG, CAPES, CNPq, PTI-Lasse e ENAP (Escola Nacional de Administração Pública) por financiarem parcialmente a pesquisa apresentada nesse artigo.

\section{Referências}

Alves, A., Morais, F., Schaab, B., and Jaques, P. (2018). Melhorando a atenção dos estudantes através da tutoria de mindfulness em sistemas tutores inteligentes. In Brazilian Symposium on Computers in Education (Simpósio Brasileiro de Informática na Educação-SBIE), volume 29, page 973.

Beiter, R., Nash, R., McCrady, M., Rhoades, D., Linscomb, M., Clarahan, M., and Sammut, S. (2015). The prevalence and correlates of depression, anxiety, and stress in a sample of college students. Journal of affective disorders, 173:90-96.

Bradley, J., Loucks, J., Macaulay, J., Noronha, A., and Wade, M. (2015). Digital vortex: How digital disruption is redefining industries. Global Center for Digital Business Transformation: An IMD and Cisco initiative. 
VIII Congresso Brasileiro de Informática na Educação (CBIE 2019)

Anais do XXX Simpósio Brasileiro de Informática na Educação (SBIE 2019)

Dantas, Á. M. C., Viana, H., Abijaude, J., and Sobreira, P. (2018). Internet das coisas e aprendizagem colaborativa: Revisão sistemática da literatura. In Brazilian Symposium on Computers in Education (Simpósio Brasileiro de Informática na Educação-SBIE), volume 29, page 278.

de Oliveira, M. A. and Duarte, Â. M. M. (2004). Controle de respostas de ansiedade em universitários em situações de exposições orais. Revista Brasileira de Terapia Comportamental e Cognitiva, 6(2):183-199.

Gaultney, J. F. (2010). The prevalence of sleep disorders in college students: impact on academic performance. Journal of American College Health, 59(2):91-97.

Hanceroglu, L. (2017). Investigating the Effects of Mindfulness on Children's Executive Function, Emotional Regulation, Stress, and Academic Performance Compared to a Control Condition. PhD thesis, Wilfrid Laurier University, https://scholars.wlu.ca/etd/1956/.

Inmon, W. H. (2016). Data Lake Architecture: Designing the Data Lake and avoiding the garbage dump. Technics Publications.

Lacerda, D. P., Dresch, A., and Antunes, J. A. V. (2015). Design Science Research: método para avanço da ciência e tecnologiaigning the Data Lake and avoiding the garbage dump. Porto Alegre, Bookman.

Lee, H. Y., Jamieson, J. P., Miu, A. S., Josephs, R. A., and Yeager, D. S. (2018). An entity theory of intelligence predicts higher cortisol levels when high school grades are declining. Child development. DOI: https://doi.org/10.1111/cdev.13116.

Lopes, V., Medina, R., Bernardi, G., and Nunes, F. B. (2018). Smart classroom utilizando dispositivos iot: uma revisão sistemática da literatura. In Simpósio Brasileiro de Informática na Educação-SBIE.

Madakam, S. (2015). Internet of things: smart things. International journal of future computer and communication, 4(4):250.

Mondardo, A. H. and Pedon, E. A. (2005). Estresse e desempenho acadêmico em estudantes universitários. Revista de Ciências Humanas, 6(6):159-180.

Mota, F., Kwecko, V., Tolêdo, F., Devincenzi, S., Ferreira, F. Z., Bicho, A., and da Costa Botelho, S. S. (2018). Metodologia e validação para a construção de gatilhos motivacionais em sistemas persuasivos computacionais para mudança de comportamento. In Simpósio Brasileiro de Informática na Educação - SBIE.

Subramaniam, B. S. (2011). Influence of body mass index on heart rate variability (hrv) in evaluating cardiac function in adolescents of a selected indian population. Italian Journal of Public Health, 8(2):149-155.

Sung, Y.-T., Chang, K.-E., and Liu, T.-C. (2016). The effects of integrating mobile devices with teaching and learning on students' learning performance: A meta-analysis and research synthesis. Computers \& Education, 94:252-275.

Zheng, H. and Genaro Motti, V. (2018). Assisting students with intellectual and developmental disabilities in inclusive education with smartwatches. In Proceedings of the 2018 CHI Conference on Human Factors in Computing Systems, page 350. ACM. 